\title{
Strong Baselines for Simple Question Answering over Knowledge Graphs with and without Neural Networks
}

\author{
Salman Mohammed, Peng Shi, and Jimmy Lin \\ David R. Cheriton School of Computer Science \\ University of Waterloo \\ smohammed1993@gmail.com, \{peng.shi,jimmylin\}@uwaterloo.ca
}

\begin{abstract}
We examine the problem of question answering over knowledge graphs, focusing on simple questions that can be answered by the lookup of a single fact. Adopting a straightforward decomposition of the problem into entity detection, entity linking, relation prediction, and evidence combination, we explore simple yet strong baselines. On the popular SimPLEQUESTIONS dataset, we find that basic LSTMs and GRUs plus a few heuristics yield accuracies that approach the state of the art, and techniques that do not use neural networks also perform reasonably well. These results show that gains from sophisticated deep learning techniques proposed in the literature are quite modest and that some previous models exhibit unnecessary complexity.
\end{abstract}

\section{Introduction}

There has been significant recent interest in simple question answering over knowledge graphs, where a natural language question such as "Where was Sasha Vujacic born?" can be answered via the lookup of a simple fact-in this case, the "place of birth" property of the entity "Sasha Vujacic". Analysis of an existing benchmark dataset (Yao, 2015) and real-world user questions (Dai et al., 2016; Ture and Jojic, 2017) show that such questions cover a broad range of users' needs.

Most recent work on the simple QA task involves increasingly complex neural network (NN) architectures that yield progressively smaller gains over the previous state of the art (see $\S 2$ for more details). Lost in this push, we argue, is an understanding of what exactly contributes to the effectiveness of a particular NN architecture. In many cases, the lack of rigorous ablation studies further compounds difficulties in interpreting results and credit assignment. To give two related examples: Melis et al. (2017) reported that standard LSTM architectures, when properly tuned, outperform some more recent models; Vaswani et al. (2017) showed that the dominant approach to sequence transduction using complex encoder-decoder networks with attention mechanisms work just as well with the attention module only, yielding networks that are far simpler and easier to train.

In line with an emerging thread of research that aims to improve empirical rigor in our field by focusing on knowledge and insights, as opposed to simply "winning" (Sculley et al., 2018), we take the approach of peeling away unnecessary complexity until we arrive at the simplest model that works well. On the SimpleQuestions dataset, we find that baseline NN architectures plus simple heuristics yield accuracies that approach the state of the art. Furthermore, we show that a combination of simple techniques that do not involve neural networks can still achieve reasonable accuracy. These results suggest that while NNs do indeed contribute to meaningful advances on this task, some models exhibit unnecessary complexity and that the best models yield at most modest gains over strong baselines.

\section{Related Work}

The problem of question answering on knowledge graphs dates back at least a decade, but the most relevant recent work in the NLP community comes from Berant et al. (2013). This thread of work focuses on semantic parsing, where a question is mapped to its logical form and then translated to a structured query, cf. (Berant and Liang, 2014; Reddy et al., 2014). However, the more recent SimpleQuestions dataset (Bordes et al., 2015) has emerged as the de facto benchmark for evaluating simple QA over knowledge graphs.

The original solution of Bordes et al. (2015) featured memory networks, but over the past several 
years, researchers have applied many $\mathrm{NN}$ architectures for tackling this problem: Golub and $\mathrm{He}$ (2016) proposed a character-level attention-based encoder-decoder framework; Dai et al. (2016) proposed a conditional probabilistic framework using BiGRUs. Lukovnikov et al. (2017) used a hierarchical word/character-level question encoder and trained a neural network in an end-to-end manner. Yin et al. (2016) applied a character-level CNN for entity linking and a separate word-level CNN with attentive max-pooling for fact selection. Yu et al. (2017) used a hierarchical residual BiLSTM for relation detection, the results of which were combined with entity linking output. These approaches can be characterized as exploiting increasingly sophisticated modeling techniques (e.g., attention, residual learning, etc.).

In this push toward complexity, we do not believe that researchers have adequately explored baselines, and thus it is unclear how much various NN techniques actually help. To this end, our work builds on Ture and Jojic (2017), who adopted a straightforward problem decomposition with simple NN models to argue that attentionbased mechanisms don't really help. We take this one step further and examine techniques that do not involve neural networks. Establishing strong baselines allows us to objectively quantify the contribution of various deep learning techniques.

\section{Approach}

We begin with minimal preprocessing on questions: downcasing and tokenizing based on the Penn TreeBank. As is common in the literature, we decompose the simple QA problem into four tasks: entity detection, entity linking, relation prediction, and evidence integration, detailed below. All our code is available open source on GitHub. ${ }^{1}$

\subsection{Entity Detection}

Given a question, the goal of entity detection is to identify the entity being queried. This is naturally formulated as a sequence labeling problem, where for each token, the task is to assign one of two tags, either ENTITY or NOTENTITY.

Recurrent Neural Networks (RNNs): The most obvious NN model for this task is to use RNNs; we examined both bi-directional LSTM and GRU variants over an input matrix comprised of word embeddings from the input question. Following

\footnotetext{
${ }^{1}$ http://buboqa.io/
}

standard practice, the representation of each token is a concatenation of the hidden states from the forward and backward passes. This representation is then passed through a linear layer, followed by batch normalization, ReLU activation, dropout, and a final layer that maps into the tag space. Note that since we're examining baselines, we do not layer a CRF on top of the BiLSTM (Lample et al., 2016; Ma and Hovy, 2016).

Conditional Random Fields (CRFs): Prior to the advent of neural techniques, CRFs represented the state of the art in sequence labeling, and therefore it makes sense to explore how well this method works. We specifically adopt the approach of Finkel et al. (2005), who used features such as word positions, POS tags, character n-grams, etc.

\subsection{Entity Linking}

The output of entity detection is a sequence of tokens representing a candidate entity. This still needs to be linked to an actual node in the knowledge graph. In Freebase, each node is denoted by a Machine Identifier, or MID. Our formulation treats this problem as fuzzy string matching and does not use neural networks.

For all the entities in the knowledge graph (Freebase), we pre-built an inverted index over $n$ grams $n \in\{1,2,3\}$ in an entity's name. At linking time, we generate all corresponding $n$-grams from the candidate entity and look them up in the inverted index for all matches. Candidate entity MIDs are retrieved from the index and appended to a list, and an early termination heuristic similar to Ture and Jojic (2017) is applied. We start with $n=3$ and if we find an exact match for an entity, we do not further consider lower-order $n$-grams, backing off otherwise. Once all candidate entities have been gathered, they are then ranked by Levenshtein Distance to the MID's canonical label.

\subsection{Relation Prediction}

The goal of relation prediction is to identify the relation being queried. We view this as classification over the entire question.

RNNs: Similar to entity detection, we explored BiLSTM and BiGRU variants. Since relation prediction is over the entire question, we base the classification decision only on the hidden states (forward and backward passes) of the final token, but otherwise the model architecture is the same as for entity detection. 
Convolutional Neural Networks (CNNs): Another natural model is to use CNNs, which have been shown to perform well for sentence classification. We adopt the model of Kim (2014), albeit slightly simplified in that we use a single static channel instead of multiple channels. Feature maps of widths two to four are applied over the input matrix comprised of input tokens transformed into word embeddings, followed by max pooling, a fully-connected layer and softmax to output the final prediction. Note this is a "vanilla" CNN without any attention mechanism.

Logistic Regression (LR): Before the advent of neural networks, the most obvious solution to sentence classification would be to apply logistic regression. We experimented with two feature sets over the questions: (1) tf-idf on unigrams and bigrams and (2) word embeddings + relation words. In (2), we averaged the word embeddings of each token in the question, and to that vector, we concatenated the one-hot vector comprised of the top 300 most frequent terms from the names of the relations (e.g., people/person/place_of_birth), which serve as the dimensions of the one-hot vector. The rationale behind this hybrid representation is to combine the advantages of word embeddings in capturing semantic similarity with the ability of one-hot vectors to clearly discriminate strong "cue" tokens in the relation names.

\subsection{Evidence Integration}

Given the top $m$ entities and $r$ relations from the previous components, the final task is to integrate evidence to arrive at a single (entity, relation) prediction. We begin by generating $m \times r$ (entity, relation) tuples whose scores are the product of their component scores. Since both entity detection/linking and relation prediction are performed independently, many combinations are meaningless (e.g., no such relation exists for an entity in the knowledge graph); these are pruned.

After pruning, we observe many scoring ties, which arise from nodes in the knowledge graph that share the exact same label, e.g., all persons with the name "Adam Smith". We break ties by favoring more "popular" entities, using the number of incoming edges to the entity in the knowledge graph (i.e., entity in-degree) as a simple proxy. We further break ties by favoring entities that have a mapping to Wikipedia, and hence are "popular". Note that these heuristics for breaking scoring ties are based on the structure of the knowledge graph, as neither of these signals are available from the surface lexical forms of the entities.

\section{Experimental Setup}

We conducted evaluations on the SimPLEQUESTIONS dataset (Bordes et al., 2015), comprised of $75.9 \mathrm{k} / 10.8 \mathrm{k} / 21.7 \mathrm{k}$ training/validation/test questions. Each question is associated with a (subject, predicate, object) triple from a Freebase subset that answers the question. The subject is given as an MID, but the dataset does not identify the entity in the question, which is needed for our formulation of entity detection. For this, we used the names file by Dai et al. (2016) to backproject the entity names onto the questions to annotate each token as either ENTITY or NotENTITY. This introduces some noise, as in some cases there are no exact matches-for these, we back off to fuzzy matching and project the entity onto the $n$-gram sequence with the smallest Levenshtein Distance to the entity name. As with previous work, we report results over the 2M-subset of Freebase.

For entity detection, we evaluate by extracting every sequence of contiguous ENTITY tags and compute precision, recall, and $\mathrm{F} 1$ against the ground truth. For both entity linking and relation prediction, we evaluate recall at $N($ R@ $N)$, i.e., whether the correct answer appears in the top $N$ results. For end-to-end evaluation, we follow the approach of Bordes et al. (2015) and mark a prediction as correct if both the entity and the relation exactly match the ground truth. The main metric is accuracy, which is equivalent to R@1.

Our models were implemented in PyTorch v0.2.0 with CUDA 8.0 running on an NVIDIA GeForce GTX 1080 GPU. GloVe embeddings (Pennington et al., 2014) of size 300 served as the input to our models. We used negative log likelihood loss to optimize model parameters using Adam, with an initial learning rate of 0.0001 . We performed random search over hyperparameters, exploring a range that is typical of NNs for NLP applications; the hyperparameters were selected based on the development set. In our final model, all LSTM and GRU hidden states sizes and MLP hidden sizes were set to 300. For the CNNs, we used a size 300 output channel. Dropout rate for the CNNs was 0.5 and 0.3 for the RNNs. For the CRF implementation, we used the Stanford NER tagger (Finkel et al., 


\begin{tabular}{lll}
\hline R@ $N$ & BiLSTM & CRF \\
\hline \hline 1 & $67.8[67.568 .0]$ & 66.6 \\
5 & $82.6[82.382 .7]$ & 81.3 \\
20 & $88.7[88.588 .8]$ & 87.4 \\
50 & $91.0[90.891 .1]$ & 89.8 \\
\hline
\end{tabular}

Table 1: Results for entity linking on the validation set, given the underlying entity detection model.

\begin{tabular}{lll}
\hline Model & R@1 & R @5 \\
\hline \hline BiGRU & $82.3[82.082 .5]$ & $95.9[95.796 .1]$ \\
CNN & $82.8[82.582 .9]$ & $95.8[95.796 .1]$ \\
LR (tf-idf) & 72.4 & 87.6 \\
LR (GloVe+rel) & 74.7 & 92.2 \\
Ture and Jojic (2017) & 81.6 & - \\
\hline
\end{tabular}

Table 2: Results for relation prediction on the validation set using different models.

2005). For LR, we used the scikit-learn package in Python. For Levenshtein Distance, we used the ratio function in the "fuzzywuzzy" Python package. Evidence integration involves crossing $m$ candidate entities with $r$ candidate relations, tuned on the validation set.

\section{Results}

We begin with results on individual components. To alleviate the effects of parameter initialization, we ran experiments with $n$ different random seeds ( $n=20$ for entity detection and $n=$ 50 for relation prediction). Following Reimers and Gurevych (2017), and due to questions about assumptions of normality, we simply report the mean as well as the minimum and maximum scores achieved in square brackets.

For entity detection, on the validation set, the BiLSTM (which outperforms the BiGRU) achieves 93.1 [92.8 93.4] F1, compared to the CRF at 90.2. Entity linking results (R@N) are shown in Table 1 for both the BiLSTM and the CRF. We see that entity linking using the CRF achieves comparable accuracy, even though the CRF performs slightly worse on entity detection alone; entity linking appears to be the bottleneck. Error analysis shows that there is a long tail of highlyambiguous entities - that is, entities in the knowledge graph that have the same label-and that even at depth 50, we are unable to identify the correct entity (MID) more than $10 \%$ of the time.

Results of relation prediction are shown in Table 2 on the validation set. Ture and Jojic (2017) conducted the same component-level evaluation, the results of which we report (but none else that

\begin{tabular}{lll}
\hline Entity & Relation & Acc. \\
\hline \hline BiLSTM & BiGRU & $74.9[74.675 .1]$ \\
BiLSTM & CNN & $74.7[74.574 .9]$ \\
BiLSTM & LR (tf-idf) & $68.3[68.268 .5]$ \\
BiLSTM & LR (GloVe+rel) & $70.9[70.871 .1]$ \\
CRF & BiGRU & $73.7[73.473 .9]$ \\
CRF & CNN & $73.6[73.473 .7]$ \\
CRF & LR (tf-idf) & 67.3 \\
CRF & LR (GloVe+rel) & 69.9 \\
\hline \hline Previous Work & \\
\hline \hline Bordes et al. (2015) & 62.7 \\
Golub and He (2016) & 70.9 \\
Lukovnikov et al. (2017) & 71.2 \\
Dai et al. (2016) & 75.7 \\
Yin et al. (2016) & 76.4 \\
Yu et al. (2017) & 77.0 \\
Ture and Jojic (2017) & 86.8 \\
\hline
\end{tabular}

Table 3: End-to-end answer accuracy on the test set with different model combinations, compared to a selection of previous results reported in the literature.

we could find). We are able to achieve slightly better accuracy. Interestingly, we see that the CNN slightly outperforms the BiGRU (which beats the BiLSTM slightly; not shown) on R@1, but both give essentially the same results for R@5. Compared to LR, it seems clear that for this task NNs form a superior solution.

Finally, end-to-end results on the test set are shown in Table 3 for various combinations of entity detection/linking and relation prediction. We found that crossing 50 candidate entities with five candidate relations works the best. To compute the [min, max] scores, we crossed 10 randomlyselected entity models with 10 relation models. The best model combination is BiLSTM (for entity detection/linking) and BiGRU (for relation prediction), which achieves an accuracy of 74.9, competitive with a cluster of recent top results. Ture and Jojic (2017) reported a much higher accuracy, but we have not been able to replicate their results (and their source code does not appear to be available online). Setting aside that work, we are two points away from the next-highest reported result in the literature.

Replacing the BiLSTM with the CRF for entity detection/linking yields 73.7 , which is only a 1.2 absolute decrease in end-to-end accuracy. Replacing the BiGRU with the CNN for relation prediction has only a tiny effect on accuracy ( 0.2 decrease at most). Results show that the baselines that don't use neural networks (CRF + LR) perform surprisingly well: combining LR (GloVe+rel) or LR (td-idf) for relation prediction 
with CRFs for entity detection/linking achieves 69.9 and 67.3, respectively. Arguably, the former still takes advantages of neural networks since it uses word embeddings, but the latter is unequivocally a "NN-free" baseline. We note that this figure is still higher than the original Bordes et al. (2015) paper. Cast in this light, our results suggest that neural networks have indeed contributed to real and meaningful improvements in the state of the art according to this benchmark dataset, but that the improvements directly attributable to neural networks are far more modest than previous papers may have led readers to believe.

One should further keep in mind an important caveat in interpreting the results in Table 3: As Reimers and Gurevych (2017) have discussed, non-determinism associated with training neural networks can yield significant differences in accuracy. Crane (2018) further demonstrated that for answer selection in question answering, a range of mundane issues such as software versions can have a significant impact on accuracy, and these effects can be larger than incremental improvements reported in the literature. We adopt the emerging best practice of reporting results from multiple trials, but this makes comparison to previous single-point results difficult.

It is worth emphasizing that all $\mathrm{NN}$ models we have examined can be characterized as "Deep Learning 101": easily within the grasp of a student after taking an intro NLP course. Yet, our strong baselines compare favorably with the state of the art. It seems that some recent models exhibit unnecessary complexity, in that they perform worse than our baseline. State-of-the-art NN architectures only improve upon our strong baselines modestly, and at the cost of introducing significant complexity-i.e., they are "doing a lot" for only limited gain. In real-world deployments, there are advantages to running simpler models even if they may perform slightly worse. Sculley et al. (2014) warned that machine-learned solutions have a tendency to incur heavy technical debt in terms of ongoing maintenance costs at the systems level. The fact that Netflix decided not to deploy the winner of the Netflix Prize (a complex ensemble of many different models) is a real-world example.

\section{Conclusions}

Moving forward, we are interested in more formally characterizing complexity-accuracy trade- offs and their relation to the amount of training data necessary to learn a model. It is perhaps self-evident that our baseline CNNs and RNNs are "less complex" than other recent models described in the literature, but how can we compare model complexity objectively in a general way? The number of model parameters provides only a rough measure, and does not capture the fact that particular arrangements of architectural elements make certain linguistic regularities much easier to learn. We seek to gain a better understanding of these tradeoffs. One concrete empirical approach is to reintroduce additional NN architectural elements in a controlled manner to isolate their contributions. With a strong baseline to build on, we believe that such studies can be executed with sufficient rigor to yield clear generalizations.

To conclude, we offer the NLP community three points of reflection: First, at least for the task of simple QA over knowledge graphs, in our rush to explore ever sophisticated deep learning techniques, we have not adequately examined simple, strong baselines in a rigorous manner. Second, it is important to consider baselines that do not involve neural networks, even though it is easy to forget that NLP existed before deep learning. Our experimental results show that, yes, deep learning is exciting and has certainly advanced the state of the art, but the actual improvements are far more modest than the literature suggests. Finally, in our collective frenzy to improve results on standard benchmarks, we may sometimes forget that the ultimate goal of science is knowledge, not owning the top entry in a leaderboard.

\section{Acknowledgments}

This research was supported by the Natural Sciences and Engineering Research Council (NSERC) of Canada.

\section{References}

Jonathan Berant, Andrew Chou, Roy Frostig, and Percy Liang. 2013. Semantic parsing on Freebase from question-answer pairs. In Proceedings of the 2013 Conference on Empirical Methods in Natural Language Processing (EMNLP 2013). Seattle, Washington, pages 1533-1544.

Jonathan Berant and Percy Liang. 2014. Semantic parsing via paraphrasing. In Proceedings of the 52nd Annual Meeting of the Association for Computational Linguistics (ACL 2014). Baltimore, Maryland, pages 1415-1425. 
Antoine Bordes, Nicolas Usunier, Sumit Chopra, and Jason Weston. 2015. Large-scale simple question answering with memory networks. arXiv: $1506.02075 v 1$.

Matt Crane. 2018. Questionable answers in question answering research: Reproducibility and variability of published results. Transactions of the Association for Computational Linguistics 6, to appear.

Zihang Dai, Lei Li, and Wei Xu. 2016. CFO: Conditional focused neural question answering with largescale knowledge bases. In Proceedings of the 54th Annual Meeting of the Association for Computational Linguistics (ACL 2016). Berlin, Germany, pages 800-810.

Jenny Rose Finkel, Trond Grenager, and Christopher Manning. 2005. Incorporating non-local information into information extraction systems by Gibbs sampling. In Proceedings of the 43rd Annual Meeting of the Association for Computational Linguistics (ACL 2005). Ann Arbor, Michigan, pages 363-370.

David Golub and Xiaodong He. 2016. Characterlevel question answering with attention. In Proceedings of the 2016 Conference on Empirical Methods in Natural Language Processing (EMNLP 2016). Austin, Texas, pages 1598-1607.

Yoon Kim. 2014. Convolutional neural networks for sentence classification. In Proceedings of the 2014 Conference on Empirical Methods in Natural Language Processing (EMNLP 2014). Doha, Qatar, pages 1746-1751.

Guillaume Lample, Miguel Ballesteros, Sandeep Subramanian, Kazuya Kawakami, and Chris Dyer. 2016. Neural architectures for named entity recognition. In Proceedings of the 15th Annual Conference of the North American Chapter of the Association for Computational Linguistics: Human Language Technologies (NAACL/HLT 2016). San Diego, California, pages 260-270.

Denis Lukovnikov, Asja Fischer, Jens Lehmann, and Sören Auer. 2017. Neural network-based question answering over knowledge graphs on word and character level. In Proceedings of the 26th International World Wide Web Conference (WWW 2017). Perth, Australia, pages 1211-1220.

Xuezhe Ma and Eduard Hovy. 2016. End-to-end sequence labeling via bi-directional LSTM-CNNsCRF. In Proceedings of the 54th Annual Meeting of the Association for Computational Linguistics (ACL 2016). Berlin, Germany, pages 1064-1074.

Gábor Melis, Chris Dyer, and Phil Blunsom. 2017. On the state of the art of evaluation in neural language models. arXiv: 1707.05589v2.

Jeffrey Pennington, Richard Socher, and Christopher D. Manning. 2014. GloVe: Global vectors for word representation. In Proceedings of the 2014 Conference on Empirical Methods in Natural
Language Processing (EMNLP 2014). Doha, Qatar, pages 1532-1543.

Siva Reddy, Mirella Lapata, and Mark Steedman. 2014. Large-scale semantic parsing without questionanswer pairs. Transactions of the Association for Computational Linguistics 2:377-392.

Nils Reimers and Iryna Gurevych. 2017. Reporting score distributions makes a difference: Performance study of LSTM-networks for sequence tagging. In Proceedings of the 2017 Conference on Empirical Methods in Natural Language Processing (EMNLP 2017). Copenhagen, Denmark, pages 338-348.

D. Sculley, Gary Holt, Daniel Golovin, Eugene Davydov, Todd Phillips, Dietmar Ebner, Vinay Chaudhary, and Michael Young. 2014. Machine learning: The high-interest credit card of technical debt. In Proceedings of the NIPS 2014 Workshop on Software Engineering for Machine Learning. Montreal, Quebec, Canada.

D. Sculley, Jasper Snoek, Alex Wiltschko, and Ali Rahimi. 2018. Winner's curse? On pace, progress, and empirical rigor. In Proceedings of the 6th International Conference on Learning Representations, Workshop Track (ICLR 2018).

Ferhan Ture and Oliver Jojic. 2017. No need to pay attention: Simple recurrent neural networks work! (for answering "simple" questions). In Proceedings of the 2017 Conference on Empirical Methods in Natural Language Processing (EMNLP 2017). Copenhagen, Denmark, pages 2856-2862.

Ashish Vaswani, Noam Shazeer, Niki Parmar, Jakob Uszkoreit, Llion Jones, Aidan N. Gomez, Lukasz Kaiser, and Illia Polosukhin. 2017. Attention is all you need. arXiv:1706.03762v5.

Xuchen Yao. 2015. Lean question answering over Freebase from scratch. In Proceedings of the 2015 Conference of the North American Chapter of the Association for Computational Linguistics: Demonstrations (NAACL/HLT 2015). Denver, Colorado, pages 66-70.

Wenpeng Yin, Mo Yu, Bing Xiang, Bowen Zhou, and Hinrich Schütze. 2016. Simple question answering by attentive convolutional neural network. In Proceedings of the 26th International Conference on Computational Linguistics (COLING 2016). Osaka, Japan, pages 1746-1756.

Mo Yu, Wenpeng Yin, Kazi Saidul Hasan, Cicero dos Santos, Bing Xiang, and Bowen Zhou. 2017. Improved neural relation detection for knowledge base question answering. In Proceedings of the 55th Annual Meeting of the Association for Computational Linguistics (ACL 2017). Vancouver, British Columbia, Canada, pages 571-581. 